\title{
Im Labyrinth - György Ligetis Étude pour piano Nr. 1 Désordre im Höranalyse-Unterricht
}

\author{
Irmgard Brockmann
}

Dieser Beitrag beschreibt ein Unterrichtskonzept im Fach Höranalyse, in dem es um die hörende Annäherung an György Ligetis erste Klavieretüde aus dem Jahr 1985 geht. Ziel der doppelstündigen Unterrichtseinheit ist die Erkenntnis wesentlicher Kompositionsprinzipien des Werks über das Hören. Im Stundenverlauf werden hierfür mehrere mithilfe einer Notationssoftware hergestellte Hörbeispiele verwendet, die sowohl getrennte Einblicke in die Parts der beiden Hände als auch in den kompositorischen Gerüstsatz des Stücks ermöglichen. Die Hörbeispiele erweisen sich nicht nur methodisch als nützlicher Kompass im polyrhythmischen Labyrinth des Werks, sondern bieten darüber hinaus auch reizvolle Analogien zu Ligetis Inspirationsquellen, und zwar sowohl zur afrikanischen Musik und den Methoden ihrer Erforschung als auch zur Maschinenästhetik der Studies for Player Piano von Conlon Nancarrow.

This article describes a lesson plan in aural analysis using György Ligeti's first piano etude from 1985. The lesson's aim is to discern essential compositional principles in Ligeti's piece through listening. The lesson uses several audio examples which have been produced through music notation software. They will render it possible to give separate insights into the use of both hands as well as into the compositional framework of the piece. The audio examples not only prove to be a methodically useful compass in the polyrhythmic labyrinth of this work, but also offer appealing analogies to Ligeti's sources of inspiration, both to African music and the methods by which African music can be studied and to the machine aesthetics in the Studies for Player Piano by Conlon Nancarrow.

SCHLAGWORTE/KEYWORDS: 20. Jahrhundert; 20th century; aural analysis; Désordre; Études pour piano; György Ligeti; Höranalyse; Lehre in Höranalyse; Methodik; methodology; teaching aural analysis

Zu Ligetis Klavieretüden und speziell zu Désordre sind bereits viele - mehr oder weniger ausführliche - analytische Untersuchungen erschienen. Während es sich hierbei durchgehend um notentextbasierte Analysen handelt, ${ }^{1}$ nähert sich der vorliegende Beitrag dem Werk auf anderem Wege: Für die Erarbeitung des Stücks im Unterricht wurde bewusst ein höranalytischer Ansatz gewählt. ${ }^{2}$ Es soll gezeigt werden, dass auch eine komplexe Kom-

$1 \quad$ Z.B. Guthrie 1989; von Loesch 1992; Kinzler 1995; Schütz 1996; Rothkamm 2004; Bouliane 2006; Batchelor 2015; Quinnett 2015. Auch die den Etüden gewidmeten Abschnitte in den Monographien von Burde, Dibelius und Floros enthalten analytische Hinweise (Burde 1993, 191-201; Dibelius 1994, 220-232; Floros 1996, 172-196).

2 Ohne eine explizite Höranalyse zu verfassen, geht Denys Bouliane - im Unterschied zu den anderen Autoren, die dies höchstens am Rande thematisieren - mehrfach auf die Wahrnehmung der analysierten Befunde beim Hören der Etüde ein. Wie wenig selbstverständlich das gleichzeitige Hören eines zu analysierenden Werks dennoch auch für ihn zu sein scheint, belegt seine im Folgenden zitierte Empfehlung: »[...] because the aural effect of the works under discussion is so important to the analysis that follows $[\ldots]$, it is highly recommended that the reader listen to the works being discussed « (Bouliane 2006, 166). 
position aus dem 20. Jahrhundert umfassend über das Gehör und ohne sofortigen Blick in den Notentext erschlossen werden kann. ${ }^{3}$

Als methodisches Hilfsmittel werden im Laufe des Unterrichts einige mit dem Notationsprogramm Sibelius hergestellte Hörbeispiele genutzt, ${ }^{4}$ die durch Reduktion ein wirkungsvolles Werkzeug für die hörende Einsicht ins Werk bieten. Die Anregung zu dieser Idee stammt von Ligeti selbst: Anlässlich der Uraufführung seines Klavierkonzerts in der revidierten Fassung erklärte der Komponist bei einem Einführungsvortrag ${ }^{5}$ wesentliche Kompositionsprinzipien seines Werks. Er ließ zunächst die verschiedenen rhythmischen Schichten des Stücks von den jeweiligen Instrumentengruppen (Streicher, Bläser, Klavier) separat und teilweise langsamer vorspielen. Das Publikum erhielt somit Einblick in einzelne Stränge der Komposition, bevor die Musiker*innen die Ausschnitte nochmals komplett und im Originaltempo vortrugen. Um ähnliche Einblicke auch für Désordre zu ermöglichen, entstand die Idee, dieses Stück mit dem Notationsprogramm abzuschreiben und anschließend verschiedene Ausschnitte und Schichten gesondert und in unterschiedlichen Tempi aufzunehmen. ${ }^{6}$

Analog zur zweiteiligen Form von Désordre verläuft auch die höranalytische Erarbeitung des Stücks im Unterricht in zwei Phasen, für die jeweils unterschiedliche methodische Ansätze gewählt wurden. In der ersten Phase, in der es um die detaillierte Höranalyse des Tonmaterials und der Kompositionstechnik geht, wird ein exploratives Verfahren ${ }^{7}$ angewandt: Die Studierenden werden durch Fragen bzw. Höraufträge schrittweise zu Erkenntnissen über die verschiedenen Schichten und Prozesse der Komposition geführt. Die dabei verwendeten Hörbeispiele beinhalten sowohl Teile der komplexen Originalkomposition als auch die mit dem Computer generierten Reduktionen, sodass abwechselnd deduktive und induktive Methoden zum Einsatz kommen. Die zweite Unterrichtsphase beginnt mit einer kreativen Aufgabe: Die Studierenden sollen eigene Ideen zur Fortsetzung des Stücks entwickeln und diese in einer Skizze festhalten. Ihre Entwürfe werden anschließend vorgestellt und gemeinsam diskutiert. Erst danach wird der zweite

3 Eine diesbezüglich extreme Position nimmt der Komponist Mauro Hertig mit seinem radikalen Plädoyer contra Partitur- und pro Höranalyse ein. Er argumentiert: »Die Aufgabe, zwischen der Partitur und dem Klang eine Brücke zu bilden, das übernehmen schon die Musiker*innen. Wir dürfen uns auf das Endprodukt der Partitur konzentrieren, das Klingende. Man kann die Noten getrost vom Heiligensessel runterholen und zurück zum Aufführungsmaterial legen« (Hertig 2019, 11).

4 Für die Herstellung der Hörbeispiele wurden Midi-Dateien aus dem Notationsprogramm Sibelius exportiert und anschließend mit den Samples eines Steinway D-Flügels aus East-West Quantum Leap Pianos (Gold) aufgenommen.

5 Rundfunkmitschnitt (29.2.1988).

6 Gerade während der Corona-Pandemie erwiesen sich die computergenerierten Hörbeispiele methodisch als hilfreich, da herkömmliche Methoden wie Mitsingen, Mitspielen oder Mitklatschen verschiedener kompositorischer Schichten im Online-Unterricht nicht realisierbar waren. - Wie sich bei abschließenden Recherchen zur vorliegenden Arbeit herausstellte, realisierte Hartmuth Kinzler eine ähnliche Idee bereits 1991 mit den Möglichkeiten der damaligen Computertechnik (vgl. Kinzler 1993). Außer der gemeinsamen Idee ergeben sich jedoch kaum Überschneidungen, da Kinzler in seinem Beitrag mehr auf die für die damalige Zeit aufwendige Herstellung des Materials als auf dessen konkrete methodische Handhabe im Unterricht eingeht. Zudem lässt sich nicht beurteilen, wie umfangreich das Klangmaterial ist, da es lediglich ausschnittsweise - in Form von im Text abgebildeten Notenbeispielen - veröffentlicht wurde.

7 Das Prinzip entspricht dem sentdeckenden Lernen« bzw. dem »Entdecken-lassenden Verfahren« (Ernst 2012, 79). 
Formteil von Désordre vorgespielt und höranalytisch untersucht. Zum Abschluss der Unterrichtsstunde erfolgt die Beschäftigung mit der Notation des Stücks, indem alle höranalytisch gewonnenen Erkenntnisse mit dem Notentext verglichen und beim Hören der Etüde mitlesend vertieft werden.

Im Folgenden wird der Verlauf der Unterrichtseinheit beschrieben, indem die jeweiligen Aufgabenstellungen zu den einzelnen Hörbeispielen sowie die entsprechenden Ergebnisse chronologisch dargestellt werden. Die vorgestellten Ergebnisse resultieren aus mehreren zum Thema durchgeführten Unterrichtsstunden. ${ }^{8}$

\section{ERSTER FORMTEIL}

Zu Beginn des Unterrichts wird das Thema der Stunde vorgestellt. Da die Unterrichtseinheit als Teil eines Ligeti-Seminars konzipiert ist, sind den Studierenden Epoche und Komponist bereits bekannt. Vor diesem Hintergrund wird ihnen als Thema eine Höranalyse zur ersten Klavieretüde von György Ligeti aus dem Jahr 1985 angekündigt, deren Titel noch nicht mitgeteilt wird.

\section{Orientierung}

Die erste Unterrichtsphase dient der globalen Orientierung im Stück. Hierfür wird der erste Formteil der Etüde (ca. 11/2 Minuten) ${ }^{9}$ zweimal vom Tonträger vorgespielt. Als Anhaltspunkt erhalten die Studierenden vor jedem Durchgang Aufgaben oder Leitfragen, die ihre Aufmerksamkeit auf bestimmte Werkaspekte lenken sollen.

Die erste Aufgabe besteht darin, den subjektiven Eindruck, den das Stück beim ersten Hören auf sie macht, in Worte zu fassen. Des Weiteren sollen sie auf den zeitlichen Verlauf der Musik achten und erwägen, ob die Musik eher statisch oder prozesshaft wirkt und welche musikalischen Parameter für die statische bzw. prozesshafte Wirkung den Ausschlag geben.

Anschließend werden die Eindrücke gesammelt. Sehr oft kommentieren die Studierenden das Gehörte spontan mit Worten wie »Chaos«, ${ }^{10}$ »Gewusel «, »Wust«, »Durcheinander « etc. Bestätigt werden diese Eindrücke durch den Titel des Stücks selbst: Désordre. Der zeitliche Verlauf wird unterschiedlich wahrgenommen: Einerseits beschreiben sie eine »mitreißende«, »zielstrebige«, »fast soghafte« Entwicklung, die sie vor allem mit dem »Höher- und Schneller-Werden der Musik« ${ }^{11}$ begründen. Andererseits nehmen sie, aufgrund der "gleichförmigen rhythmischen Bewegung u und »maschinenhaften Virtuosität» auch eine gewisse Statik wahr.

8 Beteiligt waren jeweils Studierende aus der Klassik-Abteilung der Hochschule Osnabrück (Studiengang Musikerziehung mit Hauptfach Instrumental- oder Gesangspädagogik, Elementare Musikpädagogik, Komposition oder Musiktheorie/Gehörbildung), die das Modul `Höranalyse` im 5. und 6. Semester belegen. Das hier vorgestellte Konzept beansprucht - je nach Kurszusammensetzung - in der Regel ein bis maximal eineinhalb Unterrichtseinheiten à 90 Minuten.

9 In der in meinem Unterricht verwendeten Einspielung mit Pierre-Laurent Aimard ist das Ende des ersten Formteils bei Minute 01:22 erreicht. (Die Stelle entspricht im Notentext der endgültigen Fassung Takt 101 in der rechten und Takt 97 in der linken Hand.)

10 Alle in diesem Absatz in Anführungszeichen gesetzten Beschreibungen sind Zitate von Studierenden.

11 Das Oberstimmen-orientierte (`melodische`) Hören ist den meisten Studierenden vertrauter: Dass die linke Hand im Verlauf des ersten Formteils tiefer wird, fällt ihnen beim ersten Hören oft gar nicht auf. 
Für die genauere Beurteilung ist ein weiterer Hörvorgang nötig, der unter anderem die Frage nach der pianistischen Realisation des Gehörten klären soll. Die Studierenden werden gebeten, darauf zu achten, in welchen Bereichen der Tastatur gespielt wird und inwieweit sich die Position der Hände im Laufe der Zeit verändert. Ergänzend sollen sie mitverfolgen, ob es weitere Faktoren gibt, die den zielstrebigen Entwicklungsprozess unterstützen.

Da der Hör-Fokus gezielt auf die Register des Klaviers gerichtet wurde, wird beim zweiten Hören klarer, dass sich beide Hände von der Mittellage ausgehend in die Randbereiche der Tastatur bewegen. Die sich während dieses in Richtung der Extremlagen expandierenden Prozesses aufbauende Spannung wird durch ein Crescendo verstärkt. Des Weiteren wird eine Art zeitliche Verdichtung oder Beschleunigung hörbar. Um diese näher bestimmen zu können, bedarf es zunächst einer genaueren Untersuchung und Bestimmung des der Komposition zugrundeliegenden Tonmaterials, wofür nun die bereits erwähnten, computergenerierten Hörbeispiele eingesetzt werden.

\section{Differenzierung}

\section{Tonmaterial}

Die beiden ersten Computerbeispiele präsentieren die Parts beider Hände zu Beginn des Stücks einzeln und in verlangsamtem Tempo. ${ }^{12}$ Die Studierenden erhalten die Aufgabe, auf die verschiedenen Schichten der Komposition und deren Charakteristika zu achten. Darüber hinaus soll auch die formale Struktur des Abschnitts beschrieben werden. ${ }^{13}$

(1)) https://storage.gmth.de/zgmth/media/1153/Brockmann_Hb_Audio01.wav

Hörbeispiel 1: Anfang rechte Hand; Tempo: Ganze = MM40

In diesem Tempo und in der Beschränkung auf die rechte Hand hört man unschwer zwei Schichten: eine durchlaufende Impulsfolge, bestehend aus aufwärts gerichteten Tonleiterbewegungen im (leiseren) Hintergrund und eine melodische Struktur im Vordergrund, bestehend aus akzentuierten lauteren Tönen. ${ }^{14}$ Sowohl der melodische »Vordergrund « als auch der skalare $»$ Hintergrund ${ }^{15}$ bewegen sich im siebenstufigen diatonischen Tonraum. Rhythmisch handelt es sich um eine asymmetrische Taktart: Die durchlaufenden Tonleiterbewegungen des Hintergrunds werden durch die Melodie im Vordergrund in Einheiten nach den Mustern `kurz-lang، sowie slang-kurz` gegliedert. Bei genauem Zählen wird in diesem Tempo deutlich, dass es sich um acht Werte (in diesem Fall Achtel) ${ }^{16}$ handelt, die in

12 Die mit der Notationssoftware hergestellten Hörbeispiele wurden nummeriert. Nr. 1 und 2 wurden im Tempo MM40 (ganzer Takt) aufgenommen. Das Originaltempo ist von Ligeti mit MM63 (ganzer Takt) angegeben.

13 Je nach Situation können für die Aufgabenstellungen jeweils mehrere Hörvorgänge sinnvoll bzw. nötig sein.

14 Dass es sich dabei anfangs immer um Oktaven handelt, kann - falls es nicht bewusst wahrgenommen wird - als Information mitgeteilt werden.

15 Ligeti selbst benutzt häufig dieses Vokabular: So benennt er z. B. unter der Überschrift »Taktierung, Interpretation « in den Spielanweisungen zu seinem Orchesterwerk Melodien »drei dynamische Ebenen: einen `Vordergrund`, bestehend aus Melodien bzw. kürzeren melodischen Figuren, eine mittlere Ebene, bestehend aus untergeordneten, ostinato-artigen Figurationen, und einen 'Hintergrund längeren, liegenden Tönen « (Ligeti 1973, [vii]).

16 Diese Information zur Notation wird gegeben. 
$3+5$ oder $5+3$ unterteilt werden. Außerdem lässt sich bei aufmerksamem Hinhören erkennen, dass ab und zu kleine Unregelmäßigkeiten auftreten - minimale Verkürzungen, bei denen nur sieben statt acht Achtel pro Takt erklingen. ${ }^{17}$ Formal gliedert sich die Melodie in drei Unterabschnitte, die jeweils mit einer Tonwiederholung beginnen. Dadurch entsteht eine satzähnliche Struktur (Form a a' b), die insgesamt 14 Takte umfasst $(4+4+6){ }^{18}$

Auch der Anfang der linken Hand wird separat und in verlangsamtem Tempo in der digitalen Fassung vorgespielt. Die Studierenden sollen die Unterschiede und Gemeinsamkeiten mit dem Tonmaterial der rechten Hand untersuchen.

(1)) https://storage.gmth.de/zgmth/media/1153/Brockmann_Hb_Audio02.wav

Hörbeispiel 2: Anfang linke Hand; Tempo: Ganze = MM40

Die linke Hand bewegt sich im pentatonischen Tonraum ${ }^{19}$ und spielt ebenfalls durchlaufende Achtel im Hintergrund sowie eine La-pentatonische Melodie ${ }^{20}$ in Oktaven im Vordergrund. Rhythmisch sind - wie in der rechten Hand - asymmetrisch unterteilte 8/8-Takte zu hören. Diese bleiben jedoch, anders als in der rechten Hand stets gleich lang. ${ }^{21}$ Der Abschnitt ist deutlich länger als der der rechten Hand: Bei genauer Zählung ergibt sich ebenfalls eine satzähnliche Bauweise ( $\left.\mathrm{aa}^{\prime} \mathrm{b}\right)$, diesmal bestehend aus $4+4+10=18$ Takten. $^{22}$

\section{Prozesse}

Nach der höranalytischen Erforschung des Tonmaterials sollen im nächsten Schritt die rhythmischen und melodischen Prozesse der Komposition im Detail untersucht werden. Um diese transparent zu machen, erfolgte mithilfe des Notationsprogramms die Reduktion des Stücks auf den Gerüstsatz, indem der melodische Vordergrund losgelöst vom Hintergrund abgeschrieben und somit separat hörbar gemacht wurde. Drei Hörbeispiele ${ }^{23}$ geben Einblicke in die Prozesse.

17 Diesen Effekt bezeichnet Heinz von Loesch (1992, 9) in Anlehnung an den in den Naturwissenschaften gebräuchlichen Begriff als »Dreckeffekt«: »Der anfängliche Kosmos, vergleichbar den geordneten Ausgangsbedingungen eines chemischen oder physikalischen Versuches, ist in Wahrheit gar kein Kosmos. Er ist von Beginn an nur eine Scheinordnung, erweist sich von vorneherein als 'gestörtes System`. Und die Art der Störung - das, was auf Dauer so chaotisch anmutende Konsequenzen zeigt - erinnert in auffallender Weise an die sogenannten sDreckeffektes in den Naturwissenschaften: an die kleinen, unscheinbaren Abweichungen bereits in den Ausgangsbedingungen, Unregelmäßigkeiten, die jeder Vorhersage des Wetters oder der Turbulenzen in einer Flüssigkeit und in einem Gas so problematisch machen."

18 Die hier ausformulierten Ergebnisse werden im Unterricht stichpunktartig per Beamer oder an der Tafel gesammelt.

19 Die naheliegende Vermutung, dass die linke Hand dabei ausschließlich auf schwarzen (und die rechte ausschließlich auf weißen) Tasten spielt, kann ggf. von den Absoluthörer*innen der Gruppe oder von der Lehrperson bestätigt werden.

20 Die Klassifikation pentatonischer Skalen als Do-, Re-, Mi-, So- und La-Pentatonik entspricht der Terminologie von Zsolt Gárdonyi (2002, 245-247).

21 Somit entfällt für die linke Hand der sogenannte »Dreckeffekt», vgl. oben, Anm. 17.

22 Vgl. Anm. 18.

23 Die Hörbeispiele 3-6 wurden in dem von Ligeti angegebenen Tempo (Ganze = MM 63) aufgenommen, was in etwa auch dem Tempo der Aufnahme von Pierre-Laurent Aimard entspricht. 
Zuerst erklingt der Vordergrund der rechten Hand. Die Studierenden sollen die melodischen und rhythmischen Abläufe im gesamten ersten Formteil verfolgen und dabei besonders auf die Fortsetzung der Oktavenmelodie achten.

(1)) https://storage.gmth.de/zgmth/media/1153/Brockmann_Hb_Audio03.wav

Hörbeispiel 3: Vordergrund rechte Hand, 1. Teil; Tempo: Ganze = MM63

Die Reduktion enthüllt die von den Studierenden in der Originalversion der Etüde zunächst nicht wahrgenommene Sequenzstruktur: Die oktavierte Melodie wird im weiteren Verlauf des ersten Satzes stufenweise aufwärts sequenziert. Weiterhin wird deutlich, wie der zuvor bereits beschriebene Eindruck der zeitlichen Verdichtung und Beschleunigung zustande kommt: Ab einem bestimmten Punkt verkürzt sich zunehmend der zeitliche Abstand zwischen den Melodietönen (Hörbeispiel 3 ab Minute 0:40), sodass ein auskomponiertes Accelerando entsteht. Hier lässt sich beim Hören darauf schließen, dass in der Partitur ab dieser Stelle Taktwechsel notiert werden (7/8, 6/8, 5/8 usw.). ${ }^{24}$

Anhand des nächsten Hörbeispiels soll der weitere Verlauf des melodischen Vordergrunds in der linken Hand genauer untersucht und mit den Prozessen der rechten Hand verglichen werden.

()) https://storage.gmth.de/zgmth/media/1153/Brockmann_Hb_Audio04.wav

Hörbeispiel 4: Vordergrund linke Hand, 1. Teil; Tempo: Ganze = MM 63

Die linke Hand durchläuft einen ähnlichen Prozess, jedoch abwärtsgerichtet und - wie bereits erwähnt - mit einer längeren pentatonischen Melodie: Anders als in der rechten Hand beginnt das nächste Sequenzglied jedoch nicht beim nächsttieferen Ton innerhalb der pentatonischen Skala, sondern beim jeweils übernächsten. ${ }^{25}$ Auch hier ist das auskomponierte Accelerando ab derselben Stelle deutlich hörbar (Hörbeispiel 4 ab Minute $0: 40)$.

Das nächste Computerbeispiel demonstriert das Resultat aller Abläufe im Zusammenspiel beider Hände. Die Studierenden werden gebeten, auf das Zusammenwirken der verschiedenen Prozesse zu achten und ihre Erkenntnisse schriftlich zu fixieren.

(১)) https://storage.gmth.de/zgmth/media/1153/Brockmann_Hb_Audio05.wav

Hörbeispiel 5: Vordergrund in beiden Händen, 1. Teil; Tempo: Ganze = MM63

Dem Anhören des Beispiels folgt eine Arbeitsphase, in der die Studierenden Gelegenheit haben, ihre Erkenntnisse - einzeln oder in Kleingruppen - zu formulieren. Diese werden anschließend gemeinsam besprochen und in Stichpunkten per Beamer oder an der Tafel zusammengetragen. ${ }^{26}$ Zur besseren Sortierung der komplexen Vorgänge erscheint die separate Aufteilung in Zeit- und Raumkomponenten dabei sinnvoll:

Zeitkomponenten: Die rechte Hand sequenziert eine satzähnlich aufgebaute Melodie aufwärts. Die linke Hand sequenziert eine ebenfalls satzähnliche, jedoch längere Melo-

24 Der Blick in die Partitur am Ende der Stunde bestätigt: Ligeti notiert tatsächlich kürzere Takte, obgleich er lediglich die entsprechenden Taktstriche einzeichnet und auf die Angabe von Taktarten verzichtet.

25 Die zunächst Dis-La-pentatonische Melodie wird dementsprechend mit einer Ais-Mi-pentatonischen sequenziert, danach folgen eine Fis-Do-, Cis-So-, Gis-Re-pentatonische usw.

26 Die Lehrperson kann durch geeignete Rückfragen bei der sprachlichen Präzisierung behilflich sein. 
die abwärts, sodass die Melodien den Sequenzierungsprozess nicht gleichzeitig vollziehen, sondern sich überlappen. Beide Hände spielen - anfangs für kurze Zeit synchron jeweils in einer asymmetrischen Taktart (acht Achtel, unterteilt in 3+5 bzw. 5+3). Klar getrennt ist anfangs auch der melodische Vordergrund von einem gleichmäßig bewegten Hintergrund. Durch gelegentliche Verkürzungen im Hintergrund der rechten Hand kommt es zu leichten Phasenverschiebungen zwischen rechts und links, die anfangs wie geringfügige Synchronisationsmängel wirken, sich jedoch immer mehr verschieben und schließlich als voneinander unabhängige Strukturen wahrgenommen werden. ${ }^{27}$ Durch die ab einem bestimmten Moment zusätzlich vorgenommene Stauchung des Materials entwickelt sich auf einer weiteren Ebene trotz gleichbleibenden Spieltempos ein Beschleunigungsprozess (Hörbeispiel 5 ab Minute 0:40).

Raumkomponenten: Die Anordnung der verwendeten Tonvorräte unterstützt die Teilung des Tonraums in oben und unten: Die rechte Hand spielt in der oberen Hälfte der Klaviatur ausschließlich weiße (`Weißtasten-Heptatonikı), die linke in der unteren Hälfte ausschließlich schwarze Tasten ('Schwarztasten-Pentatonikı). Im Laufe der Zeit expandiert der Raum durch das Auseinanderdriften beider Hände bis in die Randlagen des Klaviers. ${ }^{28}$ Eine weitere Raumkomponente wurde bereits beim Anhören der Originalversion der Etüde deutlich: Die Konstellation der Schichten im Sinne von Vorder- und Hintergrund vermittelt zusätzlich einen Eindruck von Raumtiefe.

Die beiden ungleich langen Melodien werden also sequenziert, gegeneinander verschoben und ab einem bestimmten Zeitpunkt zunehmend gestaucht. All diese Prozesse werden konsequent und im Crescendo bis zum Höhepunkt des Stücks weitergeführt, wo sie jeweils ihre Extreme erreichen (Randlagen der Tastatur, maximale Stauchung, fff).

Im Anschluss an diese Zusammenfassung wird nochmals das Original des ersten Teils der Etüde vom Tonträger vorgespielt. Die Studierenden sollen versuchen, alle bisher erkannten Strukturen und Prozesse nun auch in der Originalkomposition wiederzuerkennen und zu verfolgen.

\section{Fazit}

Der anfänglich zwar dichte, aber noch geordnete Eindruck geht unmerklich über in einen chaotischen Zustand - eine sehr ıordentlich komponierte Unordnung ‘. Wie in einem Labyrinth $^{29}$ verliert man im Laufe der Zeit allmählich die Orientierung, übrig bleibt als Anhaltspunkt primär der sequenzierte Melodieverlauf der rechten Hand. Die Komplexität und Mehrschichtigkeit der polyrhythmischen Abläufe bereiten beim ersten Hören oft Schwierigkeiten. Nach der ausführlichen Untersuchung des Tonmaterials und der rhyth-

27 »Whereas small phase differences over one or two beats tend to be interpreted by the ear as simply discrepancies, when three or more beats are out of sync the melodic and rhythmic structures of the two hands are likely to be construed as independent entities« (Bouliane 2006, 171).

28 Da sich der aufwärtsgehende Sequenzierungsprozess in der rechten Hand über die gesamte Etüde erstreckt, wird die oberste Lage zwar erst am Ende des Stücks erreicht; Ligeti oktaviert jedoch den Schlussklang des ersten Formteils nach oben, sodass vorübergehend die Illusion entsteht, dass auch die rechte Hand bereits an dieser Stelle den äußersten Rand der Tastatur erreicht.

29 Ligeti selbst verweist im Zusammenhang mit der Entstehung seiner Klavieretüden auf die Idee zu einer Labyrinth-ähnlichen Musik: »Ich habe mir die Aufgabe gestellt, für einen lebendigen Spieler eine ähnlich labyrinthische Musik zu schreiben, wie das Nancarrow für das mechanische Klavier tat« (Ligeti 1991, 361). 
mischen Prozesse anhand der Computerreduktionen fällt es den Studierenden jedoch erfahrungsgemäß leicht, die verschiedenen Stränge auch in der komplexen Originalkomposition zu verfolgen. Zusätzlich wird klar, dass die Stauchungen des Materials die allmähliche Auflösung des Hintergrunds zur Folge haben: Aus ursprünglich acht Achteln pro Takt werden sieben, dann sechs, fünf usw., bis sie schließlich gänzlich eliminiert sind und nur noch die Melodien im Vordergrund übrigbleiben.

\section{ZWEITER FORMTEIL}

\section{Fragment und Fortsetzung}

Die zweite Unterrichtsphase beginnt mit einer kreativen Aufgabe: Die Studierenden sollen überlegen, wie das Stück nach der erreichten Zäsur weitergehen könnte und ihre Ideen in einer Skizze festhalten. Dabei geht es nicht darum, dem Original - etwa im Sinne einer Stilkopie - möglichst nahe zu kommen, sondern vielmehr, kreativ mit dem bereits exponierten Material umzugehen und eigene Ideen zur Fortsetzung des Stücks zu entwickeln. Die kreative Beschäftigung mit dem Kompositionsprozess dient zugleich der Vorbereitung der Höranalyse: indem sich die Studierenden mit verschiedenen Möglichkeiten zur Fortsetzung des Stücks auseinandersetzen, wird ihre Aufmerksamkeit bereits auf jene kompositorischen Fragestellungen gelenkt, die im Anschluss höranalytisch untersucht werden sollen.

Die Bearbeitung der Aufgabenstellung erfolgt in Kleingruppen, in denen zuerst unterschiedliche Möglichkeiten zur Weiterführung des Stückes diskutiert werden und anschließend ein gemeinsamer Entwurf als Skizze angefertigt wird. Folgende Fragen können dabei als Leitfragen dienen: In welchen Bereichen der Tastatur soll nach der erreichten Zäsur weitergespielt werden? Inwiefern könnte das bisherige Tonmaterial variiert oder verändert werden? Wie lang soll das Stück in etwa dauern, wie viele Formteile sollen folgen? Welche Spannungskurve wäre vorstellbar und wie wird sich die Dynamik im weiteren Verlauf gestalten? Wie könnten die rhythmischen Prozesse fortgeführt oder verändert werden? Und wie soll das Stück enden?

Im Anschluss an die Gruppenarbeit werden die Studierenden gebeten, ihre Entwürfe vorzustellen. Bei der Besprechung sollen vor allem mögliche Konsequenzen ihrer Ideen bedacht werden, insbesondere solche für die Ausgewogenheit der Form, auch im Hinblick auf das Zusammenwirken der verschiedenen Parameter. Von den vielen möglichen Vorschlägen seien im Folgenden nur einige besonders exemplarische genannt.

So könnte auf den Expansionsprozess im ersten Teil ein entsprechender, zur Mitte der Tastatur führender Kontraktionsprozess folgen; alternativ ist auch die Parallelbewegung beider Hände vorstellbar. Eine weitere Idee besteht darin, dass die Hände ihr Tonmaterial tauschen, sodass die rechte Hand nach der Zäsur auf den schwarzen und die linke auf den weißen Tasten weiterspielt. Wird dieser Vorschlag von den Studierenden vorgebracht, kann sich eine Diskussion über den durch den Materialtausch der beiden Hände verursachten Farbwechsel anschließen oder können grundlegende Fragen zur Balance zwischen Kontrasten und Kontinuitäten im Formverlauf zur Sprache kommen.

Dass bei derlei Diskussionen häufig mehr Fragen aufgeworfen als Antworten gegeben werden, wird nicht als Nachteil angesehen - erklärtes Ziel ist hier nicht, das Stück zu 
Ende zu komponieren, sondern die Studierenden - auch im Hinblick auf die nachfolgende Höranalyse - für kompositorische Fragestellungen zu sensibilisieren.

Aufgrund der extremen Virtuosität von Désordre erscheint es naheliegend, dass das Stück nicht sehr lange dauern und deshalb nur ein weiterer Formteil folgen wird. Hinsichtlich der Proportionen der beiden Formteile schlagen Studierende vereinzelt vor, das Stück im Verhältnis des goldenen Schnitts, mit dem zweiten Formteil als kürzere Sektion, einzuteilen. ${ }^{30}$

Da die Spannung im ersten Teil von Désordre bereits einen Kulminationspunkt erreicht, ist es einleuchtend, das Stück im zweiten Teil ruhiger zu gestalten. Dies könnte beispielsweise durch eine Reduktion der Lautstärke oder auch - als Pendant zum ersten Formteil - durch ein auskomponiertes Ritardando realisiert werden. In Hinblick auf den Schluss ist vorstellbar, das Stück in einer extremen Klavierlage, also am unteren oder oberen Rand der Klaviatur, zu beenden. Alternativ könnte der Kontraktionsprozess beide Hände auch in der Mittellage des Klaviers zusammenführen und in einen einzelnen Ton münden lassen.

Nach der ausgiebigen Diskussion ihrer Ideen und Entwürfe sind die Studierenden in der Regel sehr gespannt auf die Originalversion des zweiten Teils, die nun vom Tonträger vorgespielt wird.

Die Eindrücke, die Ligetis Fortsetzung des Stücks bei den Studierenden hinterlässt, beziehen sich zumeist auf den Spannungsverlauf, die Atmosphäre und den Schluss der Etüde. Erfahrungsgemäß löst die geringere Ereignisdichte des zweiten Teils oft ein Gefühl von Entspannung aus: Obwohl weiterhin in hohem Tempo gespielt wird, wirkt der zweite Teil wesentlich ruhiger - ein Eindruck, den auch die im gesamten Teil eher verhaltene Dynamik mitbestimmt. Der Wechsel in die höheren Klavierregister verändert zudem die Atmosphäre des Stücks und weckt Assoziationen an impressionistische Klaviermusik. Besondere Beachtung findet - nach den vorausgegangenen kompositorischen Überlegungen - gewöhnlich der Schluss der Etüde: Die Musik verschwindet in den Hintergrund ${ }^{31}$ und löst sich am oberen Rand der Klaviatur quasi ins Nichts auf.

\section{Differenzierung}

Wie Ligeti im Einzelnen an das Tonmaterial aus dem ersten Formteil anknüpft, soll im Unterricht nun anhand des mit dem Notationsprogramm erstellten ıSkeletts tersucht werden. Da die Studierenden durch die erste Unterrichtsphase schon Übung im Umgang mit dem Tonmaterial haben, kann das separate Anhören beider Hände übersprungen und direkt mit dem Hören des zweistimmigen Gerüstsatzes begonnen werden. Die Studierenden sollen auditiv überprüfen, wie der Komponist mit dem im ersten Teil exponierten Material verfährt: Was bleibt gleich, was ändert sich? In welcher Lage wird gespielt, welche melodischen und rhythmischen Prozesse finden statt?

4)) https://storage.gmth.de/zgmth/media/1153/Brockmann_Hb_Audio06.wav

Hörbeispiel 6: Vordergrund in beiden Händen, 2. Teil; Tempo: Ganze = MM63

30 Diese Proportionierung trifft in etwa auf Désordre zu. Ligeti hat sich hierzu allerdings nicht geäußert.

31 Die die Etüde beendende Achtel-Bewegung in beiden Händen wurde nicht in das Hörbeispiel 6 integriert, da sie strukturell zum Hintergrund gehört. 
Das musikalische Material aus dem ersten Formteil wird wieder aufgegriffen und ähnlich verarbeitet: Die ungekürzten (nicht gestauchten) Melodien beider Hände bewegen sich sequenzierend auseinander, beginnen diesmal aber etwas höher, sodass insgesamt nur die obere Hälfte der Klaviatur bespielt wird. Anstelle von Oktaven erklingen jetzt zweibis vierstimmige Akkorde, die in den oberen Klavierregistern mehr Klangfülle bieten und die Aspekte der Vertikale bzw. des musikalischen Raums noch deutlicher hervortreten lassen. Rhythmisch wird nach einer längeren Periode der Synchronizität beider Hände ähnlich verfahren wie im ersten Teil: Auch diesmal treten ab einem bestimmten Moment Verschiebungen auf (Minute 0:17 in Hörbeispiel 6). Allerdings werden letztere von der linken Hand hervorgerufen, während die rechte bis zum Schluss stets gleich lange Takte spielt. Deutlich hörbar ist darüber hinaus, dass der Abstand zwischen den Akkorden der linken Hand gegen Ende hin länger wird, sodass sich anstelle von Stauchungen diesmal Dehnungen ergeben. Der so eingeleitete Entschleunigungsprozess führt zur Beruhigung und schließlich zum Ende des Stücks.

Fazit

Die Auseinandersetzung mit der sunvollendeten` Etüde verlangt von den Studierenden, die es als Musiker*innen gewohnt sind, aus ,fertigen Partituren zu musizieren, sich tiefergehend mit dem Entstehungsprozess einer Komposition und den damit verbundenen Entscheidungen zu beschäftigen. ${ }^{32}$ Auch wenn sie die Fortsetzung des Stückes nicht als Partitur notieren, sondern lediglich in ihrer Vorstellung imaginieren und eine Skizze anfertigen, finden auch in diesem Vorstadium des Komponierens bereits erste Entscheidungen und ästhetische Überlegungen in Bezug auf Proportion, Balance und Wechselwirkungen zwischen den verschiedenen Parametern statt, von deren reflektierender Erörterung die Studierenden nicht zuletzt als Interpret*innen profitieren können. Die aktive Einbindung der Studierenden in den Entstehungsprozess der Komposition wirkt sich zudem positiv auf die sich anschließende Höranalyse aus, da die Aufmerksamkeit der Studierenden durch das Experiment bereits auf spezifisch kompositorische Fragen gelenkt wurde, die im Zentrum der Höranalyse stehen.

\section{Gesamtform}

Anschließend wird die komplette Etüde nochmals in der Originalversion vorgespielt. Besonderes `Ohrenmerkı soll beim Hören nun auf die Gesamtform des Stücks gerichtet werden: Wie erlebt man die Entfaltung der Komposition im Zeitverlauf, wie wird sie im Ganzen wahrgenommen?

Bei der Beurteilung der Gesamtform des Stücks kommen unterschiedliche Aspekte zum Tragen: Zum einen grenzt sich der erste Teil mit dem zielgerichteten Beschleunigungs- und Expansionsprozess sowie der zunehmenden Lautstärke deutlich vom zweiten $\mathrm{ab}$, der zwar ebenfalls sehr stringent verläuft, aber durch die höhere Lage, reduzierte Dynamik, die ungekürzten Melodien und das auskomponierte Ritardando eine ruhigere Atmosphäre vermittelt. Zum anderen bewirken sowohl das einheitliche Tonmaterial als

32 Auch der Blick auf ein Skizzenblatt von Désordre ist für die Arbeitsweise Ligetis aufschlussreich. Eine Abbildung findet sich auf der Titelseite der Faksimile-Erstausgabe des Premier livre der Études pour piano (Ligeti 1986). 
auch das im gesamten Stück gleichbleibende Spieltempo einen homogenen Gesamteindruck und konstituieren formale Geschlossenheit. Die von den Studierenden bereits eingangs beschriebene, eigenartige Mischung von Statik und Prozesshaftigkeit erscheint ausgeglichen und wird von Ligeti selbst als stiltypisch für viele seiner Werke benannt: »Eine meiner kompositorischen Intentionen ist die Erzeugung eines illusionären musikalischen Raumes, in dem sich das, was ursprünglich Zeit und Bewegung war, als etwas Zeitloses und Unbewegliches darstellt. " ${ }^{33}$

In der abschließenden Diskussion über die Wahrnehmung der Form überwiegt im Ergebnis der Eindruck der Homogenität des Werks - ungeachtet der beschriebenen Unterschiede zwischen den beiden Formteilen wird man des Gesamtzusammenhangs und einer Vielzahl an internen formalen Verknüpfungsmöglichkeiten gewahr. Spätestens an dieser Stelle sollte ergänzend zur Sprache kommen, dass Ligeti seine Études pour piano in bewusster Anlehnung an die Tradition der europäischen Klaviermusik komponiert hat. ${ }^{34}$ Auch dieser Aspekt kann den homogenen Gesamteindruck des Stücks insofern erklären, als sich Etüden aufgrund der Beschränkung auf ein bestimmtes spieltechnisches Problem gewöhnlich durch eine einheitliche musikalische Faktur auszeichnen.

\section{Notentext}

Zum Abschluss der Unterrichtseinheit erfolgt die Beschäftigung mit dem Notentext. Die eingehende auditive Erforschung des Werks weckt erfahrungsgemäß großes Interesse an der Notation des Stücks. Eine sinnvolle Aufgabe ist jetzt, die melodischen Verläufe beider Hände in den Noten nachzuverfolgen, indem jeweils der Beginn eines jeden neuen Sequenzgliedes gekennzeichnet wird. ${ }^{35}$ Im Vergleich mit dem Notenbild konkretisieren sich die Höreindrücke: Die auditiv erarbeiteten Ergebnisse können jetzt wiederentdeckt, nachvollzogen und bestätigt werden. Ein weiterer Hörvorgang mit gleichzeitiger Notenlektüre beschließt die Stunde.

\section{SCHLUSSBEMERKUNGEN}

Entscheidende Anregungen für die Komposition der Klavieretüden und einige seiner später entstandenen Werke erhielt Ligeti durch das Studium der afrikanischen Musik südlich

33 Ligeti 2007, 294. Ähnlich äußert sich Ligeti auch an anderer Stelle, dort mit direktem Hinweis auf Désordre: »Die Grundidee der Formvorstellung ist geblieben, die sehr große Affinität zu der Vorstellung: die musikalische Zeit oder der Ablauf der Zeit ist sozusagen gefroren, es bewegt sich nicht, alles ist gleichzeitig da. Diese Konzeption der Statik ist eine Konstante bei mir. Die ist bei den rhythmisch-metrisch sehr weit differenzierten Gebilden wie gerade meine erste und sechste Klavieretüde auch da (Erwe 1986, 8).

34 Ligeti äußert sich dazu in einem Interview wie folgt: »Auch bei den Klavieretüden gibt es einiges, das in der europäischen Tradition, besonders der des 19. Jahrhunderts, fußt; und zwar meine sehr große Liebe für die Klaviermusik von Schumann und Chopin [...].«(Erwe 1986, 8) Constantin Floros (1996, 173) weist außerdem darauf hin, dass sich in den Skizzen zu Ligetis Etüden »Verweise auf Chopins Opus 10, auf Liszts Campanella und auf Debussys Pagodes« finden.

35 Nützlich kann außerdem das Abschreiben beider Melodien sein, ggf. sogar das Notieren einiger Sequenzierungen. Da sich die Intervallgrößen bei Sequenzierungen im pentatonischen Tonraum teilweise ändern (aus kleinen Terzen können große Sekunden werden und umgekehrt, aus Quarten große Terzen etc.), kann ein Notat vor allem mit Blick auf die linke Hand zur Klärung beitragen. 
der Sahara ${ }^{36}$ und durch die Beschäftigung mit den Studies for Playerpiano von Conlon Nancarrow. Damit die Studierenden den Einfluss dieser Inspirationsquellen auf Ligetis kompositorisches Denken und die Art der künstlerischen Adaption in seinem OEuvre verstehen können, sollen diese Themengebiete in der darauffolgenden Unterrichtseinheit näher betrachtet werden.

Für die Höranalyse wird als methodisches Hilfsmittel die mittels des Notationsprogramms hergestellte, auf den Vordergrund reduzierte Version des Stücks genutzt; eine solche Reduktion wäre ohne technische Hilfsmittel nicht möglich: Menschliche Interpret*innen können das 'polyphone Skelettı ohne den durchlaufenden Achtel-Hintergrund - wie in der Computerversion - schwerlich exakt spielen. Diese einkomponierte Schwierigkeit, Einzelstimmen aus dem Ganzen herauszulösen, besteht in vergleichbarer Weise auch in der afrikanischen Musik, z. B. in der »hoquetusartigen Polyphonie ${ }^{37}$ der BandaLinda, die nur in der Gemeinschaft und nicht einzeln praktiziert werden kann. ${ }^{38}$ Eine weitere Analogie zu Ligetis Inspirationsquellen ergibt sich zwischen Nancarrows Playerpiano und dem Computerklavier durch die präzise mechanische Ausführung und das entsprechende klangliche Ergebnis.

Zurückkommend auf das eingangs erwähnte Ziel der Unterrichtseinheit, nämlich die Erkenntnis wesentlicher Kompositionsprinzipien des Werks über das Hören, muss erwähnt werden, dass eine solche Erkenntnis kaum möglich wäre, hätte Ligeti - wie es der Titel suggeriert - tatsächlich beabsichtigt, in Désordre eine unstrukturierte Unordnung zu erschaffen. Angeregt durch die Chaostheorie ${ }^{39}$ interessierte ihn vielmehr, mit kompositorischen Mitteln ein kalkuliertes musikalisches Scheinchaos bzw. „Übergänge von metrischer Ordnung zu metrischer Unordnung « ${ }^{40}$ zu konstruieren. Um diese Konstruktion beim Hören von Désordre entschlüsseln und begreifen zu können, bedarf es der Höranalyse, für die sich der computergenerierte Gerüstsatz als hilfreiches Werkzeug oder - bildlich gesprochen - als nützlicher Kompass bzw. Ariadnefaden im Labyrinth der vermeintlichen Unordnung erweist.

Darüber, mit welchen Musikkulturen dieses großen geografischen Gebiets sich Ligeti eingehender befasste, gibt der Artikel »Rhythmus/Metrum/Tempo" im Lexikon Neue Musik Aufschluss, indem er auf »Ligetis Auseinandersetzung mit subsaharischer afrikanischer Musik, namentlich mit Ensembles aus Ongo-Hörnern bei den zentralafrikanischen Banda Linda, Gesängen der Aka-Pygmäen, Kiganda-Musik der Amadinda-Holmxylophone in Uganda sowie der Mbira-Lamellophonmusik der Shona in Simbabwe« verweist (Utz/Nonnenmann 2016, 534).

37 Utz $(2003,40)$ kommentiert hier ein von Simha Arom transkribiertes Initiationslied aus dem Repertoire der Ongo(-Horn)-Ensembles der Banda-Linda: „Die hoquetusartige Polyphonie entsteht durch die Verbindung der ostinaten und mit Pausen durchsetzten Perioden, die von den Musikern fortgesetzt variiert werden."

38 Ligeti bemerkt dazu: „Solche Stücke kann man nur notieren, wie Simha Arom das gemacht hat, indem er das Ganze auf Tonband aufgenommen hat, die einzelnen Musiker ihre Stimme separat mit Kopfhörern spielen ließ, so dass sie das Ganze hören, weil sie allein ihre Stimme gar nicht spielen können, und so hat er dann die verschiedenen Stimmen einzeln aufgenommen; auf diese Weise sind die Transkriptionen in Papierform entstanden« (Bouliane 1989, 58f.).

39 Vgl. von Loesch 1992 und Schütz 1996.

40 Ligeti $(2007,292)$ kommentiert: „Während in `Automne á Varsovie` die Verbindung von romantischer Hemiolentechnik und afrikanischem additivem Puls zur Erzeugung von Geschwindigkeitsillusionen eingesetzt wird, verwende ich dieselbe Verbindung in der ersten Etüde (`Désordre`), die rhythmisch vielleicht noch vertrackter ist, zur Gestaltung von Übergängen von metrischer Ordnung zu metrischer Unordnung. « 


\section{Literatur}

Batchelor, David William (2015), Gestalt Principles in Ligeti's Piano Etude sDésordres, Ph.D., University of South Carolina. https://scholarcommons.sc.edu/etd/3634 (23.12.2021)

Bouliane, Denys (1989), „Stilisierte Emotion. György Ligeti im Gespräch«, MusikTexte 28/29, 52-62.

Bouliane, Denys (2006), »Ligeti's 'Six Etudes Pour Piano Using Cultural Referents «, übers. von Anouk Lang, Theory and Practice 31, 159-161, 163-207 [zuerst erschienen als: Bouliane, Denys (2000), „Seis estudios para piano de György Ligeti«, Quodlibet: Revista de especializacion musical 17, 17-51].

Burde, Wolfgang (1993), György Ligeti: Eine Monographie, Zürich: Atlantis.

Dibelius, Ulrich (1994), György Ligeti. Eine Monographie in Essays, Mainz: Schott.

Ernst, Anselm (2012), Lehren und Lernen im Instrumentalunterricht: Ein pädagogisches Handbuch für die Praxis, Mainz: Schott.

Erwe, Hans Joachim (1986), »Interview mit György Ligeti«, Zeitschrift für Musikpädagogik 11, 3-11.

Floros, Constantin (1996), György Ligeti: Jenseits von Avantgarde und Postmoderne, Wien: Lafite.

Gárdonyi, Zsolt / Hubert Nordhoff (2002), Harmonik [1990], Wolfenbüttel: Möseler.

Guthrie, James Martin (1989), ,Etudes Pour Piano - Premier Livre, by Gyorgy [sic] Ligeti; and, 'The Song of Glory", an Original Opera in One Act, Ph.D., Louisiana State University. https://digitalcommons.Isu.edu/gradschool_disstheses/4847 (23.12.2021)

Helbing, Volker (2014), »Netzwerk, Teleologie und Diskontinuität. Zum vierten Satz aus Ligetis Klavierkonzert«, in: Musiktheorie und Vermittlung, hg. von Ralf Kubicek, Hildesheim: Olms, 303-312.

Hertig, Mauro (2019), Höranalyse - Neue Werkzeuge der musikalischen Wahrnehmung, Hofheim: Wolke.

Kinzler, Hartmuth (1993), »Musikalische Analyse und ihre Darstellung mittels kommerzieller Sequencer- und Composersoftware«, in: Neue Musiktechnologie. Vorträge und Berichte vom KlangArt-Kongreß 1991 an der Universität Osnabrück, Fachbereich Erziehungs- und Kulturwissenschaften, hg. von Bernd Enders unter Mitarbeit von Stefan Hanheide, Mainz: Schott, 301-320.

Kinzler, Hartmuth (1995), „György Ligeti. Entscheidung und Automatik in der 1 re Étude Désordre«, in: Theorie der Musik. Analyse und Deutung, Hamburger Jahrbuch für Musikwissenschaft 13, Laaber: Laaber, 337-372 [zuerst erschienen als: Kinzler, Hartmuth (1991), „György Ligeti. Decision and Automatism in `Désordre`, 1 re Étude, Premier Livre», Interface 20, 89-124].

Ligeti, György (1991), „György Ligeti über eigene Werke. Ein Gespräch mit Detlef Gojowy aus dem Jahre 1988«, in: Für György Ligeti. Die Referate des Ligeti-Kongresses Hamburg 1988, hg. von Peter Petersen, Laaber: Laaber, 349-363.

Ligeti, György (2007), Gesammelte Schriften, Bd.2, hg. von Monika Lichtenfeld, Mainz: Schott. 
Loesch, Heinz von (1992), »Eine sfraktale Seepferdchen-Etüde`. György Ligeti und die Chaosforschung", positionen. Beiträge zur neuen Musik 11, 7-12.

Quinnett, Lawrence (2014), Harmony and Counterpoint in the Ligeti Etudes, Book 1: An Analysis and Performance Guide, Ph.D., Florida State University. https://diginole.lib.fsu.edu/islandora/object/fsu:185306/datastream/PDF/view (23.12.2021)

Rothkamm, Jörg (2004), „Ordnung in der Unordnung: Neues zur Polyrhythmik in György Ligetis Klavieretüde Désordre«, Musiktheorie 19, 63-68.

Schütz, Hannes (1996), »Musik und Chaostheorie. Gedanken zu Ligetis Klavieretüde Nr. 1 - Désordre«, Musica 50/3, 170-176.

Utz, Christian (2003), „Gefrorene Turbulenz. Die Rezeption afrikanischer Musik in György Ligetis Klavierkonzert«, Neue Zeitschrift für Musik 164/3, 36-43.

Utz, Christian / Rainer Nonnenmann (2016), »Rhythmus/Tempo/Metrum«, in: Lexikon Neue Musik, hg. von Jörn Peter Hiekel und Christian Utz, Kassel: Bärenreiter, 526-541.

\section{Noten}

Ligeti, György (1973), Melodien, Faksimileausgabe, Mainz: Schott.

Ligeti, György (1986) Études pour Piano, Premier livre, vorläufige Fassung, Faksimileausgabe, Mainz: Schott.

Ligeti, György (1998), Études pour Piano, Premier livre, endgültige Fassung, komponiert 1985, Mainz: Schott.

Ligeti, György (2015), Melodien, Mainz: Schott.

\section{Aufnahmen}

György Ligeti Edition, Vol.3: Works for Piano, Klavier: Pierre-Laurent Aimard, Sony SK62308, 1996.

Rundfunkmitschnitt der Uraufführung des Klavierkonzerts (revidierte Fassung) im Wiener Konzerthaus am 29.2.1988 mit einer Werkeinführung von Ligeti, ORF Symphonieorchester, Dir.: Mario di Bonaventura, Klavier: Anthony di Bonaventura.

(C) 2021 Irmgard Brockmann (I.Brockmann@hs-osnabrueck.de)

Hochschule Osnabrück

Brockmann, Irmgard (2021), »Im Labyrinth - György Ligetis Étude pour piano Nr. 1 Désordre im HöranalyseUnterricht ", Zeitschrift der Gesellschaft für Musiktheorie 18/2, 221-234. https://doi.org/10.31751/1153

Dieser Text erscheint im Open Access und ist lizenziert unter einer

Creative Commons Namensnennung 4.0 International Lizenz.

This is an open access article licensed under a

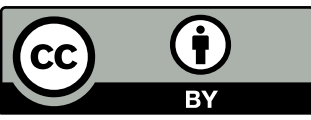

Creative Commons Attribution 4.0 International License.

eingereicht / submitted: 26/02/2021

veröffentlicht / first published: 30/12/2021

angenommen / accepted: 06/05/2021

zuletzt geändert / last updated: 23/07/2022 